\title{
Políticas públicas Y Pueblo Mapuche: BREchas Y DESAFÍOS PARA UNA AGENDA INSUFICIENTE
}

\author{
Verónica Figueroa Huencho 4
}

\section{Introducción}

La relación entre el Estado de Chile y los pueblos indígenas ha sido compleja desde el nacimiento mismo del Estado. Si bien en las últimas décadas las demandas de los pueblos indígenas han ido adquiriendo mayor notoriedad en los espacios públicos, la naturaleza de esas de- mandas es de largo aliento, y abarcan desde aspectos estructurales hasta otros de carácter político y/o administrativo que impactan en su calidad de vida y que inciden, a su vez, en la persistencia de demandas legítimas por la introducción de cambios profundos a las reglas de convivencia. Sin embargo, la solución a dichas demandas difícilmente puede ser abordada en un solo periodo de gobierno, sino que requieren de la identificación de una agenda política con mirada de largo plazo, cuestión bastante compleja de asumir como parte de compromisos a cumplir en cuatro años.

$\mathrm{Al}$ igual que años anteriores, el año 2019 estuvo marcado por decisiones de política pública que dejaron en evidencia, una vez más, las visiones sesgadas que existen al momento de definir e implementar estas políticas cuando se orientan a los pueblos indígenas, particularmente al Pueblo Mapuche, especialmente cuando se diseñan sin considerar aspectos sociales, culturales, económicos y políticos relevantes para estos pueblos. Por lo tanto, el análisis de estas decisiones no puede ser asumido solo desde una perspectiva occidental, propia de los modelos de EstadoNación, sino que requiere incorporar la mirada de los pueblos indígenas de manera de diversificar las posibilidades de avance y solución.

Entre los principales ejes temáticos presentes en la agenda de gobierno en el año 2019, podemos encontrar la continuidad en la estrategia de militarización en zonas mapuche, la aplicación de leyes especiales para resolver conflictos con comunidades mapuche, el avance en el Plan Araucanía y la política de entrega de tierras a través de lo dispuesto en el artículo 20 de la Ley № 19.253, entre otros. Sin embargo, uno de los principales temas fue la implementación de la Consulta Indígena para modificar algunos contenidos de la Ley Indígena, lo que suscitó una serie de movilizaciones que decantaron, finalmente, en la suspensión definitiva del proceso, dando

\footnotetext{
${ }^{4}$ Universidad de Chile v.figueroa.h@iap.uchile.cl
} 
cuenta de la falta de entendimiento entre los intereses de los pueblos indígenas y los del Gobierno.

En este capítulo analizaremos, desde la perspectiva de las políticas públicas, los contenidos y alcances de estas medidas impulsadas en 2019 y que permiten evidenciar elementos relevantes que tensionan la relación entre el Estado y los pueblos indígenas, pero que pueden servir de base para introducir cambios a los procesos de toma de decisiones, si existe voluntad política para ello. El foco de análisis estará puesto en el Pueblo Mapuche, pues ha sido este quien ha tenido mayor protagonismo en esta agenda. Sin embargo, las reflexiones se abordarán desde una perspectiva integral, pues las soluciones requieren cambios profundos a la institucionalidad existente en Chile para abordar la relación con los pueblos indígenas.

\section{Agenda indígena 2019: principales ejes de las políticas públicas}

De manera comparativa, no ha existido un cambio sustantivo en las políticas públicas indígenas en las últimas décadas. Más bien, las estrategias han sido de continuidad, lo que ha llevado a los gobiernos a enfrentar, de manera persistente, diferentes hechos que manifiestan el descontento de los pueblos indígenas y las limitantes que aún existen en materia de formulación e implementación de políticas. En ese sentido, el año 2019 estuvo marcado por diferentes hechos que nos permiten identificar las temáticas no resueltas y que se convierten en focos de conflicto de carácter permanente, los que abordaremos a continuación como ejes problemáticos que han requerido alguna respuesta del Estado.

\section{Militarización del territorio Mapuche}

El año 2019 comenzó con incidentes entre comunidades mapuche y organismos del Estado. Entre ellos, la toma de la municipalidad de Collipulli ${ }^{5}$ y la quema de maquinaria agrícola y de puestos de Carabineros en la provincia de Malleco $^{6}$, los que fueron atribuidos al movimiento mapuche en reclamo por el asesinato de Camilo Catrillanca, ocurrido a manos de Carabineros en noviembre de 20187 .Esta actuación de Carabineros de Chile dejó en evidencia la precariedad de los sistemas de control y fiscalización de las actuaciones de esta institución del Estado en territorio mapuche, 
así como la falta de transparencia en sus prácticas y en la aplicación de sus protocolos. De cara a la opinión pública, este hecho aumentó los niveles de desconfianza hacia la institución que ya se habían originado con la denominada "Operación Huracán" ${ }^{8}$. De hecho, el fiscal regional de La Araucanía, Cristián Paredes, daba cuenta del aumento en un 28\% de las causas por delitos vinculados a hechos de violencia rural, algunos de ellos, producto del descontento de las comunidades mapuche por el actuar de Carabineros en los dos casos mencionados. Es así como, según cifras de la Fiscalía Regional, entre noviembre (58) y diciembre (31) de 2018 se registró un tercio de los casos de violencia rural en la zona acumulados a lo largo de ese año $(33 \%)^{9}$.

Lo anterior ha servido de base para continuar con una política sostenida por los últimos gobiernos de aumentar el contingente de Carabineros y de la Policía de Investigaciones en la Región de La Araucanía. Aun cuando la Ley № 19.253 entrega al Ministerio de Desarrollo Social y Familia la coordinación de las políticas públicas de los gobiernos de turno en torno a los pueblos indígenas a través de CONADI, desde mediados del año 2000 se ha privilegiado un tratamiento de las demandas indígenas como parte de una agenda de seguridad, entregando un rol importante al Ministerio del Interior y a sus instituciones encargadas de resguardar el orden y la seguridad pública: Carabineros de Chile y Policía de Investigaciones (Figueroa, 2014). Esto ha incidido en una serie de medidas que se han intensificado en las últimas décadas, donde se ha aumentado la dotación de personal de estas instituciones en territorios ancestrales y en la denominada "zona roja", lo que se refleja tanto en las declaraciones del entonces ministro del Interior Andrés Chadwick $^{10}$, como en los $\$ 670$ millones invertidos en 11 vehículos policiales en La Araucanía a inicios del 2019 ${ }^{11}$. Se trata de una situación compleja, pues mientras se siga entendiendo que la solución es intensificar las medidas de seguridad, se continuará afectando el ejercicio de derechos legítimos de los pueblos indígenas, especialmente de aquellos que se resguardan desde el marco internacional.

Los cambios en materia de política indígena han sido graduales, pero con un claro énfasis en la intensificación de las medidas de seguridad en la Región de La Araucanía. Esto da cuenta de la visión que aún prima en las autoridades de Gobierno de distintas coaliciones, y que no ha cambiado en más de 20 años, como se refleja en las decisiones de política pública tomadas durante el año 2019.

https://www.latercera.com/nacional/noticia/fiscalia-delitos-violencia-rural-la-araucania-subieron-28/475152/

10 A raíz de diferentes ataques incendiarios atribuidos a comunidades mapuche, señalaba a los medios que "hay más de 15 personas detenidas por estas acciones y hemos ampliado y fortalecido la presencia de la PDI y Carabineros. De hecho, hay más de 208 nuevos carabineros que están redoblando la dotación frente a estos hechos de violencia; y se han iniciado acciones para recuperar terrenos tomados de forma ilegal" (https://www.google.com/search?q=gobierno+endurece+postura+en+la+araucan\%C3\%ADa+enero+2019\&oq=gobiern\&aqs=chrome. $0.69 \mathrm{i} 5912 \mathrm{j} 69 \mathrm{i} 57 \mathrm{j} 69 \mathrm{i} 59 \mathrm{j}$ 0j69i60j69i61j69i60.1551j0j4\&sourceid=chrome\&ie=UTF-8 )

11 Presentados el 3 de enero de 2019 por el director de la PDI, Héctor Espinosa. Según explicaron desde el gobierno regional de La Araucanía, "la inversión responde a las solicitudes de las juntas de vecinos, organizaciones sociales y juntas de vigilancia rural, de contar con mayor presencia en terreno de la policía civil”. (https://www.google.com/ search?q=gobierno+endurece+postura+en+la+araucan\%C3\%ADa+enero+2019\&oq=gobiern\&aqs=chrome.0.69i59l-

2j69i57j69i59j0j69i60j69i61j69i60.1551j0j4\&sourceid=chrome\&ie=UTF-8) 


\section{Aplicación de leyes especiales}

Otro eje problemático durante el año 2019 se relaciona con la continuidad en la aplicación de leyes especiales para resolver las demandas del Pueblo Mapuche. La Ley № 18.314 (o denominada "ley antiterrorista") ha sido utilizada como mecanismo para judicializar dichas demandas, de manera de disminuir la presión en la agenda política. Esta estrategia no ha dado resultados, sino que, por el contrario, ha sido fuertemente criticada por organismos de derechos humanos, tanto en Chile como a nivel internacional ${ }^{12}$.

Solo consideremos que entre 2001 y 2010 se invocó esta ley solamente para procesar a comuneros mapuche ${ }^{13}$. Sin embargo, a la fecha solo ha habido un procesamiento por esta ley, el caso de Juan Castro Antipan, quien declaró ser (desde el año 2009) un informante de Carabineros, infiltrado en el activismo mapuche tanto universitario como rural de La Araucanía. Asimismo, reconoció su participación en, a lo menos, cuatro atentados, los que fueron visados por los oficiales de inteligencia policial a quienes rendía periódicos informes en Temuco ${ }^{14}$.

En el año 2019, el Gobierno invocó esta ley antiterrorista al interponer una denuncia ante la fiscalía regional de La Araucanía por una serie de hechos violentos, entre ellos, la quema de maquinaria en el fundo San Antonio, en Lanco, y el ataque a Carabineros de Collipulli, ocurridos el martes 1 de enero. Según fuentes del Gobierno, esto respondería a un llamado hecho por diversos líderes mapuche luego de un gran encuentro en Temucuicui, en el que participaron líderes de diferentes comunidades mapuche, luego del asesinato de Camilo Catrillanca. A esto se sumó el incendio a ocho maquinarias de trabajo forestal en la comuna de Lumaco $^{15}$. En el caso de los atentados incendiarios, si bien en algunos casos no se ha podido establecer la responsabilidad, personeros del Gobierno, como el ex subsecre- tario del interior Rodrigo Ubilla ${ }^{16}$, hicieron una serie de declaraciones que contribuyeron a aumentar los prejuicios en torno al Pueblo Mapuche y sus demandas, como una forma de justificar la aplicación de estas leyes especiales.

12 Amnistía Internacional y la misma Corte Interamericana de Derechos Humanos ha señalado que la aplicación de esta ley afecta el ejercicio de derechos y viola la igualdad ante la ley de los pueblos indígenas.

13 De hecho, fue aplicada por primera vez en democracia contra los lonkos Pascual Pichun y Aniceto Norin, acusados de un ataque incendiario en los fundos San Gregorio y Nancahue, ocurridos en diciembre de 2001. También en el caso del atentado incendiario al Fundo Poluco Pidenco, de propiedad de la empresa forestal Mininco S.A., ocurrido en el mismo mes de ese año (acusando a 10 comuneros mapuche por los hechos) y a 15 mapuche acusados de asociación ilícita terrorista, los que habrían actuado bajo el amparo de la Coordinadora Arauco-Malleco, según la acusación del Ministerio Público. En este contexto se acusaba al líder de la CAM, Víctor Ancalaf, de haber perpetrado ataques incendiarios a maquinarias que desarrollaban faenas en la construcción de la Central Hidroeléctrica Ralco, hechos ocurridos el 29 de septiembre de 2001 y el 3 y 17 de marzo de 2002. Estos casos han sido solo algunos que se pueden señalar.

14 https://www.latercera.com/voces/raul-castro-antipan-el-infiltrado/

15 https://www.google.com/search?q=gobierno+endurece+postura+en+la+araucan\%C3\%ADa+enero+2019\&oq=gobierno\&aqs=chrome.1.69i59l3j69i57j0j69i60j69i61j69i60.1726j0j4\&sourceid=chrome\&ie=UTF-8

16 Con motivo de la ocurrencia de veinte incendios forestales en la Región de La Araucanía en enero de 2019, donde unas 1.699 hectáreas fueron afectadas por el fuego, el ex subsecretario del interior, Rodrigo Ubilla, señalaba a la prensa: “Yo diría que algunos de los incendios que se han producido en el último tiempo están asociados al tema de la causa mapuche” (https://www.latercera.com/politica/noticia/ rodrigo-ubilla-ministro-s-del-interior-incendios-del-ultimo-tiempo-estan-asociadosal-tema-la-causa-mapuche/532043/) 
Como lo evidencian los hechos, la aplicación de la ley antiterrorista ha sido una vía utilizada por el Estado, a través de sus diferentes gobiernos, para enfrentar las demandas políticas que tiene el Pueblo Mapuche. Efectivamente, se ha discutido en diferentes instancias respecto de la naturaleza de las demandas de este pueblo, donde el problema real tiene una base política y no de seguridad interior o de conductas terroristas. El Estado no ha estado a la altura de asumir la solución real a este problema. De hecho, en mayo de 2019 la Corte Interamericana de Derechos Humanos ordenaba al Estado de Chile a cumplir con los estándares internacionales en materia de derechos humanos en el denominado caso Norin Catriman, en donde Aniceto Norín, Pascual Pichún, Víctor Ancalaf, Florencio Marileo, Juan Marileo, José Huenchunao, Juan Millacheo y Patricia Troncoso fueron acusados bajo la ley antiterrorista, dejando sin efecto la resolución de la Corte Suprema. Este hecho es de gran relevancia, pues pone al Estado de Chile en una posición compleja frente a la opinión internacional respecto de la legitimidad en el accionar de diferentes instituciones del Estado frente a las demandas del Pueblo Mapuche ${ }^{17}$.

\section{Demandas por restitución territorial}

Las demandas del Pueblo Mapuche por restitución territorial volvieron a emerger en el año 2019, dando cuenta de la profundidad de esta problemática y de la necesidad de modificar la legislación vigente en torno a los mecanismos establecidos para la restitución de tierras. La mayoría de los hechos reseñados en el apartado anterior dicen relación con la lentitud que aún existe en la devolución de territorio ancestral, cuestión sustantiva para la mayoría de las comunidades mapuche. Una revisión de esta temática durante 2019 da cuenta de que, ya desde enero, las demandas de tierras fueron foco de conflicto al publicar la Corporación Nacional de Desarrollo Indígena (CONADI) un informe con un catastro, señalando que las reclamaciones de las comunidades mapuche se centraban en 66 predios forestales en la Región de La Araucanía, los que, a su juicio, corresponderían a casi 30 mil hectáreas ${ }^{18}$. Esto llevó a implementar una mesa de negociación entre empresas forestales, CONADI y representantes de distintas comunidades mapuche, de manera de encontrar soluciones en el marco de lo establecido en la Ley № $19.253^{19}$.

Mayores antecedentes se pueden encontrar en el capítulo de Nancy Yáñez incluido en este mismo libro.

https://www.latercera.com/nacional/noticia/la-araucania-traspaso-tierras-divide-actores-la-region/493430/

La que establece en su artículo № 20, inciso b), que se destinarán fondos para financiar mecanismos que permitan solucionar los problemas de tierras, en especial con motivo del cumplimiento de resoluciones o transacciones judiciales o extrajudiciales relativas a tierras indígenas, para dar soluciones sobre litigios de tierras indígenas o transferidas a los indígenas. Lo anterior se fin anciará con recursos aprobados año a año en la ley de presupuestos de la CONADI para el Fondo de Tierras y Aguas Indígenas (creado en esta misma ley), el que, en su aspecto central, se refiere a la solución de litigios sobre tierras entre personas indígenas, comunidades indígenas y particulares, provenientes de títulos de merced o reconocidos por títulos de comisario u otras cesiones o asignaciones hechas por el Estado a favor de los indígenas. Al año 2017, el Estado de Chile había invertido más de \$454 mil millones (USD 732 millones) en tierras (entre 1996 y 2016) en cuatro regiones del sur del país: \$354 mil millones en La Araucanía, \$48 mil millones en Biobío, \$26 mil millones en Los Ríos, \$25 mil millones en Los Lagos, y donde un 22\% de la compra de tierras se efectuó fuera de la Región de La Araucanía. De acuerdo con el registro del año 2018, a la fecha se ha entregado un total de 412 tierras a 505 familias indígenas en las regiones del Biobío, La Araucanía y Los Ríos, por un total de \$789.009.945 (ver https:// www.emol.com/noticias/Nacional/2018/01/20/891947/Costo-de-tierras-compradas-por-Conadi-para-grupos-mapu- ches-crecio-12veces-en-23-anos.html ). 
Uno de los principales problemas existentes deriva de las inconsistencias entre las cifras manejadas por las comunidades mapuche, las empresas forestales y la misma CONADI respecto a la magnitud de las tierras que deben ser restituidas a las comunidades a través de los títulos de merced. De acuerdo con las empresas forestales, la cantidad de tierras que se superponen con títulos de merced concedidos mediante leyes del siglo XIX ascienden a 3.706 hectáreas. Sin embargo, de acuerdo con las cifras de CONADI, que consideran las solicitudes ingresadas a la institución, las reclamaciones de las comunidades mapuche serían de 30.000. Finalmente, estas cifras distan de las magnitudes planteadas por las propias comunidades, quienes han señalado que estas ascienden a más de $150.000^{20}$. De acuerdo con cifras de 2019, la mayoría de las tierras reclamadas por las comunidades mapuche están en manos de empresas forestales, las que poseen más de 280 mil hectáreas de las 435 mil hectáreas que existen en la Región de La Araucanía. El holding CMPC, de acuerdo con sus propios informes, posee 170 mil hectáreas en esta región. La empresa Arauco, por su parte, posee unas 35 mil hectáreas.

CONADI, a partir de esta mesa de negociación, ha señalado que la compra de tierras, en el marco de los acuerdos que puedan surgir, debe responder a ciertos criterios ${ }^{21}$ :

- no se comprarán predios que hayan sido perturbados, que estén en toma o con historias de violencia;

- se deberá priorizar comunidades que no tengan compras anteriores; y

- se privilegiará la antigüedad de los reclamos de tierras de las comunidades²2.

Lo anterior, además, se ve presionado por los recursos de los que dispone la institución para enfrentar las demandas por compra de tierras donde, según cifras de 2019, el Fondo de Tierras y Agua disponía de $\$ 74$ mil millones, lo que obliga a adquisiciones graduales y parceladas y donde el precio promedio que se ha pagado es de $\$ 2,8$ millones por hectárea. No se debe olvidar que la especulación ha sido uno de los efectos negativos del sistema de compra de tierras. Un ejemplo: mientras que en 1994 se pagaba por los predios en conflicto un precio aproximado de $\$ 146.000$ por hectárea, al año 2000 se estaban pagando, en promedio, cerca de $\$ 1.200 .000$ por hectárea, es decir, el precio había aumentado casi seis veces (Figueroa, 2018).

En síntesis, la demanda territorial constituye un aspecto abordado de manera limitada desde las políticas públicas, especialmente por las restricciones que la misma Ley № 19.253 define en esta materia. Mientras para el Estado se trata de cuantificar espacios de tierra, principalmente porque la legislación no reconoce la existencia de "territorios indígenas", para el Pueblo Mapuche se trata de un sustento vital para la supervivencia material e inmaterial. Mientras no exista un cambio en la perspectiva con la que se valoran las culturas indígenas, o mientras exista una visión productiva de la tierra y de su explotación, seguirán existiendo inconsistencias que harán difícil la formulación de políticas.

20 https://www.google.com/search?q=las+empresas+forestales+se+sientan+a+la+mesa+2019\&oq=las+empr\&aqs=chrome.0.69i59j69i57j0l3j69i60l3.1993j0j4\&sourceid=chrome\&ie=UTF-8

21 http://www.primeranota.cl/web/region/los-predios-que-ofrecen-vender-las-empresas-forestales/

22 https://www.latercera.com/nacional/noticia/los-predios-ofrecen-vender-las-empresas-forestales/757534/ 


\section{Proceso de Consulta Indígena}

Uno de los principales ejes de las políticas indígenas en el año 2019 fue la implementación de la Consulta Indígena ${ }^{23}$, con el fin de modificar algunos aspectos de la Ley № 19.253, la que se inició el 22 de mayo de 2019, fundamentándose en lo establecido en el artículo 6 del Convenio 169 de la OIT $^{24}$. Este proceso de consulta se enmarcó, asimismo, en la política iniciada en septiembre del año 2018 denominada "Acuerdo Nacional por el Desarrollo y la Paz en La Araucanía - Plan Impulso Araucanía", la que tenía un foco principal en la Región de La Araucanía, donde se concentra gran parte de las acciones reivindicatorias de comunidades mapuche. Si bien esta política fue ampliamente criticada por no haber sido sometida a consulta, esta vez se entendió que la no implementación de ese proceso en la nueva consulta contravenía de manera grave lo estipulado en el Convenio 169 de la OIT, pues se buscaba proponer una modificación a la Ley № 19.253 que afectaría a todos los pueblos indígenas, siendo deber del Gobierno implementar una consulta a nivel nacional. Esta consulta constaba de 11 puntos que afectaban, principalmente, el artículo 13 de la Ley № 19.253, el que señala la protección de las tierras indígenas, las que "no podrán ser enajenadas, embargadas, gravadas, ni adquiridas por prescripción, salvo entre comunidades o personas indígenas de una misma etnia”. Este artículo también señala que estas tierras no podrán ser arrendadas, dadas en comodato, ni cedidas a terceros en uso, goce o administración, en el caso de que los titulares sean comunidades. Sin embargo, podrán serlo, solo por un plazo no superior a cinco años, en el caso de las de propiedad individual. Además, solo podrán permutarse por tierras de no indígenas de similar valor, con la autorización de CONADI, las que se considerarán tierras indígenas, desafectándose las primeras.

Los 11 puntos que buscaban ser sometidos a consulta en este proceso eran:

1. Posibilitar a las comunidades indígenas recibir o generar títulos individuales de dominio.

2. Permitir que el derecho real de uso y derecho real de goce puedan constituirse en título individual de dominio.

3. Eliminar la restricción de subdivisión mínima de tres hectáreas, aplicándose las reglas generales de subdivisión.

Liderada por el Ministerio de Desarrollo Social y Familia, a cargo del ministro Alfredo Moreno.

Ésta establece la obligatoriedad de los gobiernos a: i) consultar a los pueblos interesados, mediante procedimientos apropiados y, en particular, a través de sus instituciones representativas, cada vez que se prevean medidas legislativas o administrativas susceptibles de afectarles directamente; ii) establecer los medios a través de los cuales los pueblos interesados puedan participar libremente, por lo menos en la misma medida que otros sectores de la población y a todos los niveles, en la adopción de decisiones en instituciones electivas y organismos administrativos y de otra índole responsables de políticas y programas que les conciernan; y iii) establecer los medios para el pleno desarrollo de las instituciones e iniciativas de esos pueblos y, en los casos apropiados, proporcionar los recursos necesarios para este fin". 
4. Permitir la venta de tierras indígenas entre indígenas a partir de los cinco años contados desde su entrega.

5. Reglamentar el mecanismo de permuta de tierras indígenas para clarificar y precisar sus condiciones.

6. Permitir la realización de contratos de arriendos, comodatos o medierías en tierras indígenas por un plazo de hasta 25 años.

7. Permitir mecanismos alternativos y voluntarios de reparación a los problemas de tierras indígenas.

8. Permitir el acceso a beneficios asociados a la calidad indígena solamente a personas con calidad indígena adquiridas por el artículo 2, letras a) y b) de la Ley $\mathrm{N}^{\circ} 19.253$.

9. Mayores requisitos para la constitución de nuevas comunidades indígenas.

10. Permitir que las asociaciones indígenas puedan ser constituidas por un mínimo de dos integrantes.

11. Permitir que las asociaciones indígenas puedan postular al Fondo de Desarrollo Indígena.

Sin embargo, desde un inicio, este proceso fue rechazado por la mayoría de los pueblos indígenas, impidiendo su realización, negándose a participar o tomando por la fuerza los recintos destinados a la realización de los talleres. Entre los fundamentos para ello se encontraban que no se contaba con toda la información para participar del proceso, las propuestas del ejecutivo no estaban disponibles en la página de la consulta para poder ser revisadas y no existían garantías de que los acuerdos o consentimientos logrados fueran vinculantes, es decir, que fueran respetados por el Estado al someter la modificación de la ley al trámite legislativo de rigor ${ }^{25}$.

Entre las principales críticas que se hicieron es que la mayoría de estas propuestas se centraban en lo económico, donde las primeras siete medidas se referían a los títulos de dominio de tierras indígenas y la posibilidad de arrendarlas, propiciando el debilitamiento de la propiedad colectiva y favoreciendo la inversión económica de terceros no indígenas. Por otra parte, se argumentó que las medidas proponían una división de la propiedad de la tierra que sería entregada a título personal, lo que contraviene la cosmovisión de los pueblos indígenas. En ese contexto, la Asociación de Municipalidades con Alcalde Mapuche (AMCAM), luego de una serie de incidentes entre comuneros de distintas localidades de las regiones del Biobío y La Araucanía con funcionarios del Gobierno a cargo de la consulta, pidió su suspensión señalando que "la realización del proceso de consulta en un clima hostil evidencia la polarización que genera y que su contenido no ha sido socializado debidamente con las comunidades" ${ }^{26}$.

https://www.noticiaslosrios.cl/2019/05/28/que-es-la-consulta-indigena-y-por-que-su-rechazo/

https://www.latercera.com/nacional/noticia/la-consulta-indigena-los-pueblos-no-quieren/696810/

Finalmente, debido a todos estos problemas, en noviembre de 2019, el nuevo ministro de Desarrollo Social, Sebastián Sichel, anunció la suspensión definitiva del proceso de consulta a través de una minuta interna ${ }^{27}$. Lo anterior da cuenta de la necesidad de considerar los derechos 


\section{Capitulo 1. Página 25}

consagrados en la normativa internacional respecto de la obligatoriedad de los Estados de consultar a los pueblos indígenas las medidas que les afecten. En ese sentido, si bien Chile ha ratificado el Convenio 169 de la OIT en el año 2008, los procesos de consulta implementados a la fecha han sido criticados por las comunidades por no cumplir con los estándares apropiados, la buena fe y la disponibilidad de información. Por lo tanto, las políticas públicas deben adecuarse a estos requerimientos no solo como una obligación, sino como un marco para avanzar hacia una institucionalidad que no contravenga los derechos de estos pueblos.

\section{Avances, desafíos y brechas de las políticas indígenas en Chile}

En el caso de Chile, los pueblos indígenas se han convertido en un importante actor social y político que ha presionado por cambios en las políticas públicas y por el reconocimiento efectivo de sus derechos, muchos de los cuales han sido recogidos en pactos y convenios internacionales ratificados por el Estado de Chile en las últimas décadas. El análisis de las principales problemáticas surgidas en el año 2019 permite evidenciar que la política indígena continúa siendo un ámbito problemático en las agendas de los gobiernos, donde no ha existido voluntad o capacidad para comprender el verdadero alcance de sus demandas, las que provienen de un sector de la población que no solo representa culturas diferenciadas, sino que, además, está compuesta por sujetos de derechos específicos. De esa manera, mientras no existan avances sustantivos en esta materia, las reclamaciones de estos pueblos continuarán, especialmente porque no se respetan sus derechos legítimos, cuestión que ha sido visibilizada por diferentes organismos internacionales.

Si bien la promulgación de la Ley № 19.253 en el año 1993 significó un importante hito al establecer normas específicas sobre protección, fomento y desarrollo de los indígenas y al crear la institucionalidad encargada de la implementación de las políticas indígenas, la CONADI (vigente hasta nuestros días), no ha implicado cambios sustantivos en el reconocimiento de derechos políticos. En efecto, el Estado de Chile no ha reconocido constitucionalmente a los pueblos indígenas que se encuentran en su territorio. Tampoco existe el estatus jurídico de "pueblo" para ellos, pues la ley vigente les entrega el estatus de etnias, limitando el ejercicio de derechos recogidos en las normas internacionales, entre otros elementos. Todo esto ha incidido en la situación de marginación y exclusión que aún siguen experimentando.

Donde señalaba que "habiéndose analizado la oportunidad y conveniencia de continuar adelante con el procedimiento de Consulta Indígena antes mencionado, y en virtud del artículo 61 de la Ley $\mathrm{N}^{\circ} 19.880$, que establece las bases de los procedimientos administrativos que rigen los actos de los órganos de la administración del Estado, que permite la revocación de los actos administrativos a los órganos que los hubieren dictado, esta secretaría de Estado ha decidido revocar la Resolución Exenta º 241, de 2019, del Ministerio de Desarrollo Social, poniendo término al procedimiento de Consulta Indígena iniciado en el mes de abril del presente año" (https://www.latercera.com/politica/noticia/gobierno-cancela-definitivamente-consulta-indigena-medio-crisis-adportas-conmemoracion-muerte-catrillanca/899770/). Mayores antecedentes sobre este proceso de consulta y la conflictividad desatada se pueden encontrar en el capítulo de Víctor Tricot y German Bidegaín incluido en este mismo libro. 
De acuerdo al Censo 2017, 12,8\% de la población del país se considera perteneciente a algún pueblo indígena, de los cuáles 79,8\% se reconoció como mapuche, 7,2\% aymara, 4,1\% diaguita, $1,5 \%$ quechua, $1,4 \%$ lickanantay, $0,9 \%$ colla, $0,4 \%$ rapa nui, $0,2 \%$ kawésqar y $0,1 \%$ yagán. En el caso de la pobreza indígena, la Encuesta CASEN 2015 mostró que el 18,3\% de la población indígena es pobre, mientras que dentro de la población no indígena, esta cifra corresponde a un 11\%. Relacionado con lo anterior, otros estudios dan cuenta del empobrecimiento de estos pueblos, especialmente en las denominadas Áreas de Desarrollo Indígena (la mayoría ubicadas en la Región de La Araucanía), donde la pobreza de la población mapuche es de un 24,34 \%. Más recientemente, esta misma encuesta aplicada en el año 2017, indicó que 19,8\% de la población en territorio chileno era pobre multidimensionalmente, mientras que entre el Pueblo Mapuche esta cifra ascendía a 30,8\%.

Respecto de las lenguas indígenas, si bien desde el año 1996 existe un Programa de Educación Intercultural Bilingüe, existe una pérdida progresiva de estas. Un 78,6\% de la población indígena no habla ni entiende su lengua, siendo esto particularmente grave en los menores de 14 años, donde un 88,6\% se encuentra en esa condición (CASEN 2015). En salud, por su parte, un estudio del Ministerio de Salud (MINSAL) de 2016, muestra tasas mayores de mortalidad infantil en población indígena a partir de una muestra de 11 consultorios en diferentes comunas con alta población indígena.

Las problemáticas que enfrentan los pueblos indígenas son multidimensionales y resulta de particular atención la que vive el Pueblo Mapuche. Este pueblo no solo es mayoritario, sino que ha protagonizado la mayoría de las acciones de reivindicación que han tenido como respuesta acciones sesgadas desde los espacios de política pública. Son finalmente sus comunidades las principales destinatarias de las decisiones de militarización del territorio, de aplicación de leyes especiales y de proyectos de extracción que afectan el territorio y sus ecosistemas, entre otros. Es por ello que la política indígena ha tendido, en las últimas décadas, a focalizarse en este pueblo, derivando también en un enfoque restringido respecto de la propia diversidad que tienen los pueblos indígenas. En este sentido, el análisis de las brechas y los desafíos que aún persisten en la política indígena deben ser abordados desde una perspectiva sistémica, que considere aquellas demandas de carácter estructural que implicarían cambios profundos al modelo de Estado actual y a la distribución y ejercicio del poder, hasta otras que se encuentran en el ámbito de la institucionalidad política necesaria para representar de mejor forma los intereses de estos pueblos. Y finalmente, aquellas demandas que se encuentran en un nivel administrativo, principalmente, la generación de programas o proyectos específicos, y que han sido el foco de las acciones de política pública en los últimos años. A continuación, analizaremos estas tres dimensiones (estructural, política y administrativa) de manera de aportar elementos para una discusión en torno a los temas pendientes, pero también en aquellas temáticas que deberían ser incorporadas a la agenda nacional.

\section{Brechas y desafíos en el ámbito estructural}

Las movilizaciones surgidas desde el 18 de octubre del año 2019 no solo evidenciaron las desigualdades que existen en nuestra sociedad, sino también aquellas que de manera específica 


\section{Capitulo 1. Página 27}

han afectado y afectan a los pueblos indígenas. Es así como desde la marcha masiva del 25 de octubre, hasta otras que se fueron produciendo en fechas posteriores, la bandera mapuche tuvo un protagonismo importante, convirtiéndose en un símbolo de resistencia no solo del Pueblo Mapuche, sino como una forma de representar el descontento de toda la sociedad chilena ${ }^{28}$. De esa manera, la discusión en torno a la reforma constitucional también incorporó la dimensión de las demandas de los pueblos indígenas, donde la idea de avanzar hacia un Estado plurinacional se ha convertido en un ámbito que ha concitado gran interés.

Como ha expresado el historiador Fernando Pairican "la república de Chile vive un momento constituyente. Como en pocas ocasiones, la posibilidad de desmantelar el republicanismo homogéneo ha develado la oportunidad de pensar en un nuevo tipo de Estado que aspire a que las naciones originarias sean portadoras y sujetos de derecho al interior de la democracia chilena" ${ }^{29}$. Es que, por siglos, el modelo de Estado-Nación ha privilegiado la prevalencia de una cultura occidental chilena por sobre los pueblos indígenas. Esto sirvió de base para la consolidación de un proyecto de nación homogénea y para la generación de mecanismos uniformes y centralizados de participación y representación de los intereses ciudadanos. De esa manera, no solo se configuró un espacio estandarizado de interrelaciones, sino que se consolidó una visión negativa de lo indígena, siendo asociado a la pre-modernidad y al subdesarrollo, donde su forma de vida no concordaba con los ideales de la nación que se buscaba instalar (Figueroa, 2014). Esto dio lugar a un diseño de Estado y de sociedad sin los pueblos indígenas, donde la igualdad sirvió de sustento para la homogeneización de los sistemas de representación política, pero también para la utilización y ocupación de los espacios públicos y de poder.

En Chile, la idea de una sola nación ha significado la adopción de un solo modelo cultural, jurídico, político e incluso (hasta antes de 1925) religioso, y que se tradujo en una concepción centralista del ejercicio del poder. El reconocimiento de la plurinacionalidad del Estado es un ámbito estructural que implicaría un cambio sustantivo en la forma en la que se formulan e implementan las políticas de pueblos indígenas en nuestro país. Sin embargo, no ha sido objeto de atención de los actores políticos en las últimas décadas. En el año 2018, el senador Francisco Huenchumilla presentó un proyecto de reforma constitucional para el reconocimiento de la plurinacionalidad en Chile, el que no tuvo el apoyo necesario para avanzar en el poder legislativo, siendo una de las propuestas más acabadas que se han presentado a la fecha ${ }^{30}$. Ningún Gobierno ha incluido en sus programas o iniciativas la posibilidad de avanzar hacia un Estado plurinacional, siendo una demanda planteada más bien desde la ciudadanía, por lo que cabrá esperar el desarrollo del proceso constituyente para evaluar cómo avanzará esta demanda.

\footnotetext{
https://interferencia.cl/articulos/el-protagonismo-de-la-bandera-mapuche-en-la-gran-marcha-un-simbolo-politico-de-las https://ciperchile.cl/2019/12/20/estado-plurinacional-el-debate-mapuche-actual/

"Proyecto de reforma constitucional que reconoce la plurinacionalidad y los derechos de los pueblos indígenas" (Boletín N $\left.{ }^{\circ} 11.873-07\right)$.
} 
Relacionado con lo anterior, también se encuentra el reconocimiento constitucional a los pueblos indígenas, cuestión que no ha sido considerada en ninguna de las Constituciones que el Estado de Chile ha tenido hasta ahora. En el año 2015, a partir del proceso constituyente llevado a cabo en el Gobierno de la presidenta Michelle Bachelet (2014-2018), se realizaron una serie de encuentros específicos para los pueblos indígenas, cuyos resultados mostraban las demandas más relevantes para ellos: cambio constitucional con reconocimiento de los pueblos originarios, la definición del Estado chileno como uno plurinacional y el respeto a los tratados internacionales ratificados por Chile, así como la no aplicación de la ley antiterrorista ${ }^{31}$. De esa forma es posible evidenciar que las problemáticas que surgieron durante el año 2019 responden a demandas insatisfechas y sentidas de la población indígena, entre ellas, el Pueblo Mapuche.

La propuesta de reforma constitucional para el reconocimiento de los pueblos indígenas que surgió de este proceso fue claramente insuficiente. En ella se señalaba que el Estado se comprometía a respetar la integridad, derechos y cultura de los pueblos indígenas y reconocía las distintas formas de educación indígena. Sin embargo, no se hacía referencia a la libre determinación, autonomía, derechos territoriales o la plurinacionalidad, entendiendo a los pueblos indígenas como parte de la nación chilena. Desde esa fecha, no se ha avanzado en esta materia, y la agenda del Gobierno actual tampoco se ha hecho cargo de esta demanda. En una reunión sostenida en La Moneda el 14 mayo de 2019, con diputados y senadores de diferentes partidos, el presidente Sebastián Piñera señalaba que "estamos conversando para encontrar acuerdos para incorporar el reconocimiento constitucional de nuestros pueblos originarios en nuestra Constitución" y que "este es un tema, un problema y una oportunidad; se arrastran durante décadas y siglos, estamos conscientes.

Por eso, no podemos dejar pasar un minuto más"32. Por otra parte, en la cuenta pública del año 2019, el presidente señalaba que impulsará "el reconocimiento constitucional de nuestros pueblos originarios, sin debilitar la unidad de nuestra nación"33. Tal como se ha señalado, no ha habido ningún avance. El reconocimiento constitucional podrá ser discutido e incorporado en la discusión en torno a la nueva Constitución que se llevará adelante este año, pero se trata de una cuestión aún incierta.

Lo anterior se relaciona, asimismo, con otros derechos importantes para el Pueblo Mapuche que han estado presentes en los TRAWÜN (encuentros) realizados en el año 2019, donde destaca el realizado el 2 de noviembre en el sector de Kowilew con comunidades mapuche de Temuco, con la participación de diferentes líderes y lideresas de estos territorios, quienes acordaron los puntos mínimos a ser recogidos en una nueva Constitución. Entre ellos:

https://www.biobiochile.cl/noticias/nacional/region-de-los-lagos/2017/06/06/presentan-los-resultados-del-proceso- constituyenteindigena-desarrollado-en-osorno.shtml

2 https://prensa.presidencia.cl/discurso.aspx?id=95630

33 https://www.biobiochile.cl/noticias/nacional/region-de-la-araucania/2019/06/01/la-araucania-pinera-reitera-firmeza- contraterrorismo-y-mas-reconocimiento-a-pueblos-originarios.shtml 
a) Nueva Constitución plurinacional, donde se reconozca a las múltiples naciones que componen este Estado.

b) Reconocimiento histórico del despojo e invasión del territorio mapuche.

c) Derecho al autogobierno y autonomía.

d) Retiro de las forestales en Wallmapu.

e) Educación mapuche y administración propia. f) Escaños reservados para el Pueblo Mapuche.

g) Desmilitarización del Wallmapu.

h) Que los convenios internacionales y procesos de consulta y participación sean vinculantes.

Estas demandas son coincidentes con aquellas que se han planteado a lo largo de las últimas décadas por el Pueblo Mapuche y su movimiento social, y que supone el reconocimiento de derechos culturales, económicos y lingüísticos, entre otros.

De las demandas señaladas hay dos que también emergieron con fuerza durante el año 2019 y que no han sido abordadas desde las políticas públicas. Una de ellas es el derecho a la autonomía y a la libre determinación. La libre determinación como derecho humano se consagra en dos pactos internacionales de derechos humanos fundamentales, adoptados por la ONU en 1966: el Pacto Internacional de Derechos Civiles y Políticos y el Pacto Internacional de Derechos Económicos, Sociales y Culturales, además del Convenio 169 de la OIT. Todos, instrumentos ratificados por el Estado de Chile. En ese sentido, el nuevo proceso constituyente también permite repensar cómo ejercer este derecho desde la realidad que tienen los pueblos indígenas en la actualidad, donde producto del despojo territorial se nos ha limitado la idea de una real autonomía.

El otro es el de los escaños reservados, especialmente en la representación en el Congreso, de manera de asegurar la participación de los pueblos indígenas en el proceso legislativo a través de mecanismos que respeten sus propias institucionalidades ancestrales y de elección de representantes. De hecho, en una encuesta realizada por el Laboratorio Constitucional de la Universidad Diego Portales en marzo de 2019, un 66\% de los encuestados favorecía la idea de establecer escaños reservados para pueblos indígenas en el Congreso. Sin embargo, dado que esa alternativa no avanzó, el proceso de reforma constitucional ha permitido que se discuta la disponibilidad de escaños reservados en la integración de la Convención Constituyente. Este proyecto fue aprobado el 19 de diciembre en la Cámara de Diputados sin acuerdo respecto de la proporcionalidad de estos ${ }^{34}$.

El análisis de esta dimensión estructural resulta, por tanto, importante para señalar que es aquí donde debe existir una voluntad real del Estado por avanzar en el reconocimiento de los pueblos indígenas, pues se convierte en un paraguas fundamental que orientará la formulación e implementación de políticas públicas. Sin embargo, dado que se trata de cambios profundos que requieren, a su vez, de cambios culturales de mediano y largo aliento, hasta el día de hoy se carece de voluntad política por parte de los tomadores de decisión por focalizar acciones en este ámbito.

34 Por su parte, el 20 de enero de 2020, la Comisión de Constitución, legislación, justicia y reglamento del Senado aprobó la idea de legislar el proyecto de reforma constitucional para reservar "escaños a representantes de los pueblos originarios en la integración del órgano constituyente que se conforme para la creación de una nueva Constitución política”, lo que aún no ha sido aprobado por la Sala del Senado. Ver https://www.senado.cl/respaldo-unanime-a-reforma-que-establece-escanos-reservados-parapueblos/senado/2020-01-20/152304.html 


\section{Brechas y desafíos en el ámbito político-institucional}

El ámbito de la institucionalidad es otra dimensión donde las políticas públicas indígenas han sido insuficientes, lo que podría atribuirse a las limitantes estructurales a las que hacíamos mención anteriormente. Esto incide en las posibilidades de innovar en este ámbito, donde han sido los ideales de la república y de la democracia liberal los privilegiados para la definición de las normas y leyes que han regido la convivencia social y, por tanto, también a los pueblos indígenas. Dado que no existe reconocimiento constitucional, los derechos que buscan hacer exigibles los pueblos indígenas se ven obstaculizados. El reconocimiento de la existencia de una sola nación y la afirmación de que la soberanía recae en ella, ha servido para limitar la búsqueda de sistemas de participación que consideren las prácticas territoriales, culturales o políticas particulares de estos pueblos, en particular del Pueblo Mapuche.

Por otra parte, Chile se ha caracterizado por ser un Estado unitario, con un alto nivel de centralismo político y con una concentración importante de poder a nivel presidencial, el que posee atribuciones legislativas y poderes exclusivos (Mainwaring y Shugart, 2002). Esa centralización del poder ha limitado el desarrollo de normas que permitan un reconocimiento apropiado de los pueblos indígenas, buscando, más bien, su asimilación a la nación chilena. Tampoco han existido sistemas federados o descentralizados de ejercicio del poder que permitan el desarrollo de algún nivel de autonomía territorial para este pueblo.

Entre las principales limitantes en este ámbito podemos encontrar el escaso avance en materia de institucionalidad indígena y reconocimiento de derechos. La Ley № 19.253 de 1993 que establece normas sobre protección, fomento y desarrollo de los indígenas no reconoce la existencia de pueblos indígenas u originarios con preexistencia al Estado de Chile. Si bien en su artículo 1 señala que "El Estado reconoce que los indígenas de Chile son los descendientes de las agrupaciones humanas que existen en el territorio nacional desde tiempos precolombinos, que conservan manifestaciones étnicas y culturales propias siendo para ellos la tierra el fundamento principal de su existencia y cultura", a continuación indica que estas tendrán el carácter de "etnias", diferenciadas cultural y lingüísticamente respecto de la población nacional, pero sin un estatus legal que reconozca sus derechos en cuanto pueblo. Con ello se desconocen una serie de derechos que ampara la normativa internacional y que incidirá en aspectos como la autodeterminación, el autogobierno, así como el ejercicio de derechos políticos y de representación, los que se ven limitados en una política de este tipo. La ley, más bien, entiende a estas "etnias" como grupos a los que se debe proteger sin darles un lugar estratégico en definiciones de carácter político. Es así como la ley, en su artículo 1, inciso 3, señala que "es deber de la sociedad en general, y del Estado en particular, respetar, proteger y promover el desarrollo de los indígenas, sus culturas, familias y comunidades".

Junto con lo anterior, la Ley № 19.253 creó la Corporación Nacional de Desarrollo Indígena (CONADI) como un organismo público dependiente del Ministerio de Desarrollo Social y Familia, encargado de promover, coordinar y ejecutar la acción del Estado en favor del desarrollo integral de las personas y comunidades indígenas en lo económico, social y cultural, así como de impulsar su participación en la vida nacional. De esa forma, se generó una figura compleja, pues no se crea una institución que formule políticas públicas, sino una que solo actúa como ente coordinador de las decisiones de los gobiernos de turno. La formulación queda depositada en diferentes ministerios 


\section{Capitulo 1. Página 31}

sectoriales con dificultades para identificar las demandas de estos pueblos, pues no son destinatarios directos de sus respectivos ministerios.

El principal desafío en este ámbito es avanzar en la creación de institucionalidad que permita, al menos, fortalecer la centralización en la formulación de políticas públicas indígenas, a través de la creación del Ministerio de Pueblos Indígenas y el Consejo de Pueblos indígenas, ambos aún en discusión en el Congreso Nacional.

En enero del 2016 se ingresó el proyecto de ley que crea el Ministerio de Pueblos Indígenas ${ }^{35}$ al Senado $^{36}$, sin embargo, a la fecha este proyecto no ha avanzado, encontrándose actualmente en segundo trámite constitucional en el Senado. Si bien en su discurso de cuenta anual del año 2019, el presidente Sebastián Piñera señalaba que un pilar de su Gobierno sería "reconocer, valorar y promover la cultura, historia, tradiciones, lenguaje y cosmovisión de nuestros pueblos originarios, a través de la creación del Ministerio de Pueblos Indígenas y el Consejo de Pueblos Indígenas, y una consulta para perfeccionar la Ley Indígena", luego de cuatro años de discusión legislativa esto no se ha concretado.

Misma suerte ha corrido la creación del “Consejo de los Pueblos Indígenas”37, ingresado a la Cámara de Diputados el 11 de enero de $2016^{38}$, encontrándose actualmente en su segundo trámite constitucional en el Senado. En términos generales se busca crear consejos de cada uno de los nueve pueblos indígenas, como "entidades autónomas, representativas, participativas y de consulta" ante los organismos del Estado, los que se regirán por ley y por normas internas, que deberán conservar los valores tradicionales de cada pueblo. Además, establece la creación del “Consejo Nacional de Pueblos Indígenas", entidad autónoma, participativa y de consulta, que abordará materias que afecten a todos los pueblos indígenas.

Por lo tanto, el marco legal y normativo de los pueblos indígenas requiere una actualización a la luz de los avances que existen en esta materia en el ámbito internacional, resguardado el derecho a consulta de estos pueblos y propendiendo a la creación de espacios de representación apropiados a democracias modernas, caracterizadas por la diversidad de sus sociedades. Se hace urgente, asimismo, avanzar en la creación de institucionalidad que permita formular políticas públicas indígenas a través de un ministerio que cuente con las prerrogativas para ello, que pueda centralizar la toma de decisiones y actuar al más alto nivel político.

El proyecto de ley señala que su objetivo es crear "una Secretaría de Estado encargada de colaborar con el(la) Presidente(a) de la República, en el diseño, coordinación y evaluación de las políticas, planes y programas destinados a promover y fortalecer los derechos de los pueblos indígenas, su desarrollo económico, social y cultural, procurar la eliminación de toda forma de discriminación arbitraria contra los pueblos, comunidades y personas indígenas".

36 A través del boletín № 10.525-06 a la Comisión de Gobierno, Descentralización y Regionalización. Sin embargo, la discusión sobre la nueva institucionalidad estuvo cuatro meses detenida sin tramitación, lo que llevó al Gobierno a retirar del Senado la iniciativa para poder reponerla en la Cámara, lo cual finalmente realiza el día 17 de mayo de 2016, a través del boletín № 10.687-06.

37 Tal como se señala en el proyecto, su objetivo es "crear un Consejo Nacional de los Pueblos Indígenas y los Consejos de Pueblos, para efectos de generar instancias de representación de los intereses, las necesidades y los derechos colectivos de los pueblos indígenas ante los organismos del Estado".

38 A través del boletín $\mathrm{N}^{\circ}$ 10526-06. 


\section{Capitulo 1. Página 32}

\section{Brechas y desafíos en el ámbito administrativo}

Finalmente, es posible evidenciar que la mayoría de los esfuerzos de los gobiernos han estado focalizados en el ámbito administrativo, especialmente a través de la adecuación de ciertos espacios dentro de la misma burocracia pública con planes o programas específicos para los pueblos indígenas. Iniciativas que, finalmente, no tienen un efecto importante en la promoción de nuevas formas de interrelación o de posicionamiento de estos pueblos en los espacios de toma de decisiones. Se trata, por lo tanto, de uno de los ámbitos con mayor desarrollo en las últimas décadas porque no significa, necesariamente, costos políticos.

Como se ha señalado, el año 2019 marcó la continuidad de la política "Acuerdo Nacional por el Desarrollo y la Paz en La Araucanía - Plan Impulso Araucanía”, donde existió un énfasis en el ámbito económico que buscaba el aumento de la inversión privada y pública en la Región de La Araucanía. Este plan, anunciado en 2018, consideraba una cartera inicial de 491 proyectos de inversión pública a desarrollarse en un periodo de ocho años. Esta cartera de proyectos de inversión equivale a un aporte de cerca de USD 8.043 millones para el periodo 2018 a 2026, es decir, un promedio de inversión de USD 894 millones por año. Solo para el año 2019, este esfuerzo de priorización implicaría un incremento de 35\% de los recursos dispuestos para inversión en la región respecto del año 2018, pasando de USD 695 millones a USD 940 millones.

En mayo de 2019, en la presentación de la agenda legislativa indígena, el presidente Sebastián Piñera señalaba que el Plan Araucanía había permitido el crecimiento de la región "en un 6,5\%, creó 12.500 nuevos empleos y logró reducir la tasa de victimización", contemplando "una inversión de 8.200 millones de dólares que va a permitir a La Araucanía recuperar el tiempo perdido, y ojalá ponerse a la vanguardia del desarrollo de nuestras regiones"39. Por su parte, en la cuenta pública del año 2019, señalaba que se continuaría profundizando en la implementación de este plan, fortaleciendo la capacidad de desarrollo, creación de empleos e infraestructura económica y social en La Araucanía, donde el Plan Impulso Araucanía habría "permitido a esta región pasar de un crecimiento de 2,8\% en 2017, a 6,5\% en 2018, y reducir su tasa de desempleo de $8,8 \%$ a $6,2 \% " 40$.

Una de las principales críticas que se han hecho a este tipo de iniciativas no solo es su sesgo economicista, sino que restringe el análisis de la realidad de la política indígena a indicadores cuantitativos, tratándolo solo como un tema de pobreza y no como un tema de derechos. En ese sentido, este plan fue criticado por no haber sido sometido a consulta, de acuerdo con los estándares del Convenio 169 de la OIT, entendiendo que, al tratarse de una medida centrada en la gestión de recursos, no tenía un impacto en el ejercicio de derechos legítimos. Asimismo, la implementación del Plan Araucanía demuestra la necesidad de coordinación en materia de gestión, así como una vinculación permanente entre los niveles políticos y administrativos, pues de los primeros depende la aprobación oportuna de recursos.

https://prensa.presidencia.cl/discurso.aspx?id=95630

https://www.biobiochile.cl/noticias/nacional/region-de-la-araucania/2019/06/01/la-araucania-pinera-reitera-firmeza-contraterrorismo-y-mas-reconocimiento-a-pueblos-originarios.shtml 


\section{Capitulo 1. Página 33}

De hecho, desde el propio oficialismo surgieron críticas por el escaso avance en la ejecución de presupuestos vinculados al Plan Araucanía, donde no han existido avances adecuados en el ámbito legislativo ${ }^{41}$. También este Plan tiene una limitante estructural al entender que el desarrollo de los pueblos indígenas solo se centra en la Región de La Araucanía. Si bien, de acuerdo con la encuesta CASEN 2017, es una de las regiones más pobres de Chile, la problemática indígena es de carácter sistémico y requiere de una acción del Gobierno que incorpore a los demás pueblos.

Finalmente, es importante señalar que este plan contemplaba una medida compleja como era el establecimiento de un proceso de regularización de títulos en la región, lo que incide en la elaboración de catastros de tierra. Por esa vía se esperaba conocer con certeza la magnitud de las tierras reclamadas por las comunidades y, en función de eso, estimar la "deuda" pendiente, pues un problema para CONADI ha sido que las comunidades, producto de sus sistemas de herencia $\mathrm{u}$ otros, se subdividen y esto lleva a multiplicar la demanda de predios. De acuerdo con algunos organismos de derechos humanos, esta medida podría vulnerar los derechos sobre el territorio de los pueblos indígenas consagrados en el Convenio 169 de la OIT y la Declaración sobre Pueblos Indígenas.

Otra dimensión administrativa en la que se debe avanzar es en la modernización de CONADI. Si bien el proyecto de ley que crea el Ministerio de Pueblos Indígenas contempla la creación de un Servicio Nacional de Pueblos Indígenas, que vendría a reemplazar a la actual CONADI, mientras este proyecto de ley no avance, será este organismo el encargado de coordinar los esfuerzos de los gobiernos en la implementación de políticas indígenas, sin atribuciones políticas. Dentro de los programas más complejos se encuentra el Fondo de Tierras y Agua, el que requiere de una revisión, especialmente, por las limitaciones que tiene para resolver los problemas de demandas de restitución territorial. El sustento de este fondo, destinado a resolver estas demandas vía compra de terrenos o de entrega de subsidios, no considera dimensiones políticas o culturales relevantes. Además, el rol asignado al mercado en la compra de tierras por parte de CONADI ha dado lugar a la especulación, como ya se ha señalado.

Por otra parte, es necesario dotar a CONADI de recursos de diferente tipo para afrontar diferentes dimensiones de las demandas territoriales. Por ejemplo, la autorización de compras de tierras indígenas por personas no indígenas ha puesto nuevamente en tela de juicio la efectividad de CONADI para fiscalizar este procedimiento, relevando la dimensión política de estas decisiones. Entre ellas, el caso más bullado ha sido la compra de tierras por parte del ex subsecretario del Interior Rodrigo Ubilla, quien enfrenta una demanda por una presunta compra de tierras indígenas, lo que sería ilegal de acuerdo con la normativa actual ${ }^{42}$.

41 https://www.biobiochile.cl/noticias/nacional/region-de-la-araucania/2019/09/22/a-casi-un-ano-del-anuncio-del-plan-

araucaniadiputados-critican-baja-ejecucion-y-estancamiento.shtml

42 Esta demanda se originó a partir de hechos ocurridos en 2009 cuando Ubilla adquirió cuatro predios al interior de la comunidad Mariano Millahual, en Pucón. La compraventa se efectuó entre el entonces subsecretario y Guadalupe Moris Gajardo tras la liquidación de la sociedad conyugal que había mantenido esta última con Jorge Painequir. Esto dejó en evidencia la existencia de resquicios para adquirir tierras indígenas fuera de lo estipulado en la Ley № 19.253. (https:// www.latercera.com/nacional/noticia/subsecretario-ubilladeclara-denuncia-compra-irregular-tierras-indigenas/909824/) 


\section{Capitulo 1. Página 34}

Finalmente, mientras no se avance en materia institucional y se continúen generando programas para pueblos indígenas, una cuestión a resolver será la coordinación interinstitucional. Una investigación del Centro de Estudios Públicos (CEP), en el año 2019, daba cuenta de la falta de coordinación y coherencia entre los programas indígenas desarrollados desde diferentes instituciones públicas, donde la mayoría surge por cuestiones coyunturales más que planificadas, siendo sujetos de constantes modificaciones por parte de las autoridades. Asimismo, en el proceso de diseño no se tiene claridad sobre los potenciales beneficiarios, abundan los problemas de gestión (acentuados por los constantes cambios) y no se cuenta con mediciones de eficacia y calidad de los programas (Aninat y Hernando, 2019).

\section{Conclusiones}

En este capítulo buscamos abordar el análisis de la realidad de los pueblos indígenas desde un enfoque de política pública, con foco en los problemas que ha debido enfrentar el Pueblo Mapuche durante el año 2019, y que da cuenta de las limitantes que aún existen en los procesos de formulación e implementación de las políticas públicas para estos pueblos. De esa forma, surgen desafíos a las agendas gubernamentales donde es necesario abordar la relación del Estado con los pueblos indígenas de manera sistémica, diferenciándola de otras políticas públicas al tratarse de una parte de la población con derechos específicos que no ha sido adecuadamente reconocida en el caso de Chile.

Los ejes analizados muestran la persistencia en el tiempo de áreas que se hace necesario abordar de manera estructural. Sin embargo, esto requiere de enfoques de política que consideren a los pueblos indígenas como actores sustantivos del proceso. Los resultados del proceso de consulta implementado el año 2019, así como aspectos del Plan Araucanía o de las demandas territoriales, entre otros, demuestran los sesgos y el desconocimiento respecto de los pueblos indígenas, de su historia, de sus derechos y de sus culturas.

Por otra parte, queda en evidencia que el tratamiento sectorial de la política indígena no constituye un enfoque apropiado. Se trata de un ámbito de política pública con incidencia en otros de carácter económico, educativo, sanitario y social, entre otros. En ese sentido, el análisis presentado reafirma la necesidad de cambios estructurales que incorporen marcos de formulación desde nuevos paradigmas para con los pueblos indígenas, considerando sus conocimientos, epistemologías, formas de representación, etc. Mientras se siga privilegiando la mirada desde la seguridad, con protagonismo del Ministerio del Interior, será difícil establecer confianzas que permitan avanzar hacia cambios reales. Estamos, por tanto, frente a una agenda insuficiente e inconclusa. 


\section{Bibliografía}

FIGUEROA HUENCHO, V. (2014). Formulación de políticas públicas indígenas en Chile. Santiago: Editorial Universitaria

(2018). "Tierras y territorios indígenas: dimensiones complejas para las políticas públicas". Serie Policy Papers CIIR, 2.

MAINWARING, S., y SHUGART, M. (2002). Presidencialismo y democracia en América Latina. Buenos Aires: Paidós.

\section{Cronología}

\begin{tabular}{|c|c|c|}
\hline Fecha & Acontecimiento & Descripción \\
\hline $\begin{array}{l}1 \text { de enero } \\
\text { de } 2019\end{array}$ & $\begin{array}{l}\text { Ataques incendiarios en La } \\
\text { Araucanía }\end{array}$ & $\begin{array}{l}\text { Quema de maquinaria en el fundo San Antonio, en } \\
\text { Lanco, y el ataque a Carabineros de punto fijo en } \\
\text { Collipulli. }\end{array}$ \\
\hline $\begin{array}{l}2 \text { de enero } \\
\text { de } 2019\end{array}$ & $\begin{array}{l}\text { Gobierno se querella por ley de } \\
\text { seguridad del Estado }\end{array}$ & $\begin{array}{l}\text { eEl gobierno, a través de la Intendencia de La } \\
\text { Araucanía presentó una denuncia a la fiscalía } \\
\text { regional, contra quie nes resulten responsables } \\
\text { de incitación a la violencia por varios hechos. }\end{array}$ \\
\hline $\begin{array}{l}4 \text { de } \\
\text { de } 20\end{array}$ & $\begin{array}{l}\text { Gobierno impulsa mayor presencia } \\
\text { de efectivos de la PDI en La } \\
\text { Araucanía }\end{array}$ & $\begin{array}{l}\text { aLa policía civil aumentará dotación investigativa } \\
\text { aen la zona, tras últimos hechos de violencia. }\end{array}$ \\
\hline $\begin{array}{l}10 \text { de } \\
\text { enero de } \\
2019 \\
\end{array}$ & $\begin{array}{l}\text { Desalojo de Municipio de } \\
\text { Collipulli }\end{array}$ & $\begin{array}{l}\text { La sede se encontraba tomada por un grupo de } \\
\text { comuneros mapuche con varias demandas, entre } \\
\text { ellas el asesinato de Camilo Catrillanca. }\end{array}$ \\
\hline $\begin{array}{l}20 \text { de } \\
\text { enero de } \\
2019 \\
\end{array}$ & $\begin{array}{l}\text { Instalación de mesa por traspaso } \\
\text { de tierras }\end{array}$ & $\begin{array}{l}\text { oForestales, comunidades mapuche y CONADI } \\
\text { buscan definir las magnitudes de las demandas } \\
\text { territoriales. }\end{array}$ \\
\hline $\begin{array}{l}14 \text { de } \\
\text { mayo de } \\
2019\end{array}$ & Agenda legislativa indígena & $\begin{array}{l}\text { Presidente Piñera presenta la agenda legislativa } \\
\text { indígena en La Moneda. }\end{array}$ \\
\hline $\begin{array}{l}16 \text { de } \\
\text { mayo de } \\
2019 \\
\end{array}$ & Caso Norín- & $\begin{array}{l}\text { Corte Suprema deja sin efecto las condenas, } \\
\text { siguiendo lo planteado por la Corte } \\
\text { Interamericana de derechos Humanos. }\end{array}$ \\
\hline $\begin{array}{l}22 \text { de } \\
\text { mayo de } \\
2019 \\
\end{array}$ & Inicio de Consulta Indígena & $\begin{array}{l}\text { El Ministerio de Desarrollo Social y Familia inicia } \\
\text { el proceso de Consulta Indígena que busca } \\
\text { modificar aspectos de la Ley } 19.253 \text {. }\end{array}$ \\
\hline $\begin{array}{l}13 \text { de } \\
\text { noviembre } \\
\text { de } 2019\end{array}$ & Sus & $\begin{array}{l}\text { Debido al rechazo de las comunidades } \\
\text { indígenas el Ministerio de Desarrollo Social y } \\
\text { Familia decide suspender definitivamente la } \\
\text { Consulta Indígena. }\end{array}$ \\
\hline
\end{tabular}

